\title{
Metformin Treatment Prevents Sedentariness Related Damages in Mice
}

\author{
Pamela Senesi, ${ }^{1,2}$ Anna Montesano, ${ }^{1}$ Livio Luzi, ${ }^{1,2}$ Roberto Codella, ${ }^{1,2}$ \\ Stefano Benedini, ${ }^{1,2}$ and Ileana Terruzzi ${ }^{3}$ \\ ${ }^{1}$ Department of Biomedical Sciences for Health, University of Milan, Milan, Italy \\ ${ }^{2}$ Metabolism Research Center, San Donato Hospital and Scientific Institute, Milan, Italy \\ ${ }^{3}$ Diabetes Research Institute, Metabolism, Nutrigenomics and Cellular Differentiation Unit, \\ San Raffaele Scientific Institute, Milan, Italy \\ Correspondence should be addressed to Ileana Terruzzi; terruzzi.ileana@hsr.it
}

Received 25 September 2014; Revised 1 April 2015; Accepted 20 May 2015

Academic Editor: Bagher Larijani

Copyright (C) 2016 Pamela Senesi et al. This is an open access article distributed under the Creative Commons Attribution License, which permits unrestricted use, distribution, and reproduction in any medium, provided the original work is properly cited.

\begin{abstract}
Metformin (METF), historical antihyperglycemic drug, is a likely candidate for lifespan extension, treatment and prevention of sedentariness damages, insulin resistance, and obesity. Skeletal muscle is a highly adaptable tissue, capable of hypertrophy response to resistance training and of regeneration after damage. Aims of this work were to investigate METF ability to prevent sedentariness damage and to enhance skeletal muscle function. Sedentary 12-week-old C57BL/6 mice were treated with METF ( $250 \mathrm{mg} / \mathrm{kg} \mathrm{per} \mathrm{day,}$ in drinking water) for 60 days. METF role on skeletal muscle differentiation was studied in vitro using murine C2C12 myoblasts. Muscular performance evaluation revealed that METF enhanced mice physical performance (Estimated $\mathrm{VO}_{2 \max }$ ). Biochemical analyses of hepatic and muscular tissues indicated that in liver METF increased AMPK and CAMKII signaling. In contrast, METF inactivated ERKs, the principal kinases involved in hepatic stress. In skeletal muscle, METF activated AKT, key kinase in skeletal muscle mass maintenance. In in vitro studies, METF did not modify the $\mathrm{C} 2 \mathrm{C} 12$ proliferation capacity, while it positively influenced the differentiation process and myotube maturation. In conclusion, our novel results suggest that METF has a positive action not only on the promotion of healthy aging but also on the prevention of sedentariness damages.
\end{abstract}

\section{Introduction}

Type 2 diabetes mellitus is a metabolic disorder characterized by chronic hyperglycemia in association with insulin resistance, impaired relative and/or absolute insulin production, and altered glucagon secretion $[1,2]$. At the onset of diabetes two main processes are involved in its pathogenesis: progressive decline in pancreatic islets function and reduced insulin sensitivity in peripheral tissues [3]. In particular, insulin resistance (IR) occurs when insulin effect on muscle and fat tissues glucose uptake is defective and is not capable of inhibiting endogenous glucose production by the liver [4]. Because skeletal muscle is responsible for $70 \%-80 \%$ of total insulin-stimulated glucose uptake, skeletal muscle IR is a major determinant of type 2 diabetes [5].
Interactions between genetic and environmental factors, overnutrition, and sedentary behavior promote the progression and pathogenesis of IR. In particular, the modifications that occurred in the global food system during the past 3-4 decades have created an "obesogenic" environment contributing to the increase of the obesity epidemic and consequent IR incidence increase. Unhealthy diet and physical inactivity are considered among leading causes of the same diseases characterized by IR. Currently, alleviating this condition is still one of the key strategies to treat $[2,6]$.

METF, a widely prescribed drug in type 2 diabetes, is being increasingly considered for treatment and prevention of sedentariness damages, as well as for the extension of healthy lifespan [7]. Recent data showed that long-term diet supplementation with METF extends healthy lifespan in $C$. 
elegans and in middle-aged male mice $[8,9]$. In addition, our group demonstrated how acute METF treatment may induce the generation of neohypertrophic myotubes, by using an in vitro model of satellite cells (C2C12 cells line) [10].

Regular practice of physical exercise plays a very important role in maintaining a good state of health and physical well-being [11]. In particular, recent publications showed the active function of exercise in the reduction and counteraction of the mechanisms underlying muscle atrophy and degeneration related to the onset of peripheral IR $[12,13]$.

In exercising muscle, increased energy metabolism and ATP production is obtained by an increased glucose utilization. One of the most relevant metabolic effects of exercise is the enhancement of insulin action [14]. Many factors may contribute to increasing insulin sensitivity induced by exercise: a reduction in fat mass, an increase in muscle mass, and the increase of membrane-bond glucose transporters (GLUT4) in muscle cells [15].

The effects of physical exercise may have relevant implications in the prevention and treatment of metabolic diseases. In fact, by increasing insulin sensitivity, physical activity can reduce the risk of pathological conditions such as type 2 diabetes and metabolic syndrome [16].

Understanding the complex mechanisms that regulate insulin response and the onset of peripheral insulin resistance represents a primary goal in the treatment of diabetes and obesity complications, particularly by targeting skeletal muscle. Given the growing prevalence of the disease and the conditions of relative sarcopenia related to it, new therapeutic interventions are able not only to reduce the loss of skeletal muscle mass but also to stimulate muscle regeneration while preserving the physiology of viable muscle satellite cells become necessary.

In order to determine if Metformin could relieve the sedentariness damages, we studied METF effects in sedentary adult young mice, focusing our attention on METF ability to maintain mouse physical performance during submaximal incremental test.

\section{Research Design and Methods}

2.1. Materials. Mouse $\mathrm{C} 2 \mathrm{C} 12$ myoblasts were purchased from the European Collection of Animal Cell Cultures (ECACC). Reagents were purchased from Sigma Chemical Co. (Saint Louis, MO, USA). Primary antibodies against AKT (C-20), CAMKII (M-176), calnexin (H-70), ERK1 (K-23), ERK2 (C-14), GAPDH (FL-335), MyoD (C-20) Myogenin (D-10), MyHC (H-300), Myf5 (c-20), N-cadherin (H-63), p70S6 (C-18), SOD2 (FL-222), pERK1/2 (E-4-4) pp70S6 (sc-7984), peroxidase-conjugated secondary antibodies for Western blot analysis, and rhodamine-conjugated antibodies for immunofluorescence analysis were purchased from Santa Cruz Biotechnology (Santa Cruz, CA, USA). Primary antibodies phospho-AKT (Ser473) (D9E) XP and phosphoAMPK alpha (Thr172) (40H9) were purchased from Cell Signaling Technology (Danvers, MA, USA). Antibody against Phalloidin (Alexa Fluor 488 Phalloidin, molecular probesInvitrogen).

\subsection{Animal Studies}

2.2.1. Animals. Male C57BL/6 mice $(n=10)$, purchased from Charles River Laboratories (Boston, MA, USA), were used for the study at 12 weeks of age. All animals were kept on a $12 \mathrm{~h} / 12 \mathrm{~h}$ light/dark cycle with unlimited access to standard rodent chow food and water. Mice were divided into two paired groups: one treated with METF and the other not treated (CONTR). Every week weight and blood glucose levels were determined. Blood glucose was measured in blood collected from the tip of the tail with a portable glucose measuring device (Bayer, Basel, Switzerland).

2.2.2. METF Treatment. METF (Sigma Chemical Co., Saint Louis, MO, USA) was added to the drinking water at dose of $250 \mathrm{mg} / \mathrm{kg}$ body weight per day, for 60 days. Control mice received water without METF. As reported in literature [17], our pilot study confirmed that C57BL/6 mice consumed $7 \mathrm{~mL}$ water per day; METF addition did not influence water consumption. Water and METF were changed daily and the dose adjusted to weight gain each week (Figures 1(a) and 1(b)).

2.2.3. Muscular Performance Exercise Test. Muscular performance exercise was evaluated by a submaximal incremental test prior to and upon completion of the study. Briefly, mice were placed in adapted treadmill (Columbus Instruments) for $5 \mathrm{~min}$ at a $0^{\circ}$ incline, and then treadmill speed was increased according to the scheme shown in Figure 1(a) with 1.5-min intervals at a $15^{\circ}$ incline (Figure 1(a)). Animals run until exhaustion, which is defined as remaining on the shocker plate for more than 5 seconds. Food and water were unavailable to mice during any running sessions [18]. At the end of experiment, mice were sacrificed and tissues were harvested, frozen in liquid nitrogen, and stored at $-80^{\circ} \mathrm{C}$ for further analyses.

This study was conducted in compliance with approved institutional animal care of the University of Milan.

2.3. Cell Culture Experimental Procedures. C2C12 cells were maintained at $37^{\circ} \mathrm{C}$ in humidified $5 \% \quad \mathrm{CO}_{2}$ atmosphere in a growth medium (GM) containing DMEM (Dulbecco Modified Eagle Medium) supplemented with 20\% (v/v) fetal bovine serum (FBS), 1\% penicillin streptomycin, and 1\% 1glutamine up to $70 \%$ confluence. Cell differentiation was initiated by placing $70 \%$ confluent cell cultures in differentiation medium (DM), containing DMEM supplemented with $1 \%$ horse serum (HS), antibiotics, and 1\% l-glutamine. In our in vitro differentiation model, early myotubes appeared 24-48 hours (h) after serum starvation and neomyotubes formation was completed after $72 \mathrm{~h}$ [19]. Proliferating cells, myoblasts during differentiation process, and neomyotubes were treated with $400 \mu \mathrm{m}$ METF. In the control cells METF was not added to medium. Figure 2 explains experimental study design in each phase of the protocol, with cell confluence percentage, treatments start time, and duration. 


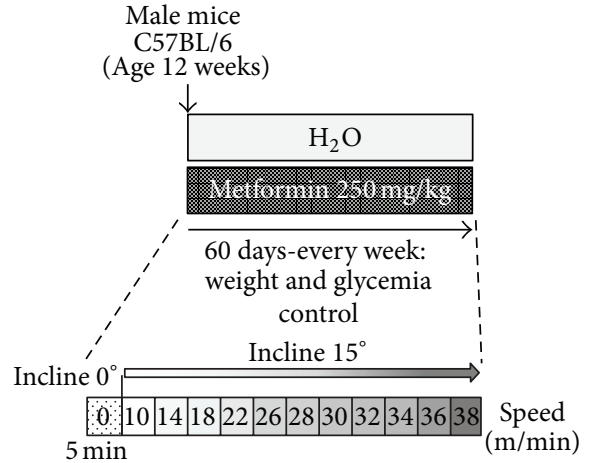

(a)
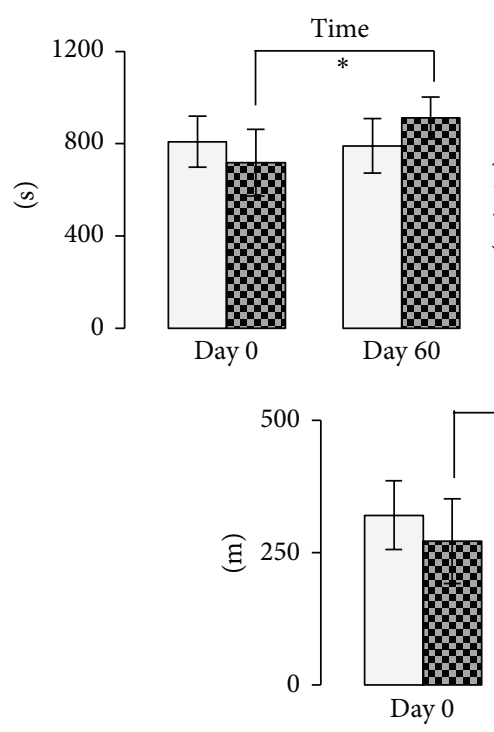

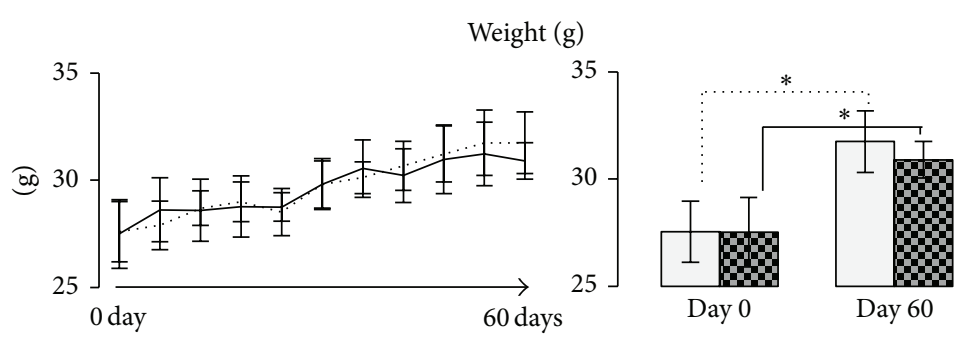

Glycemia (mg/dL)
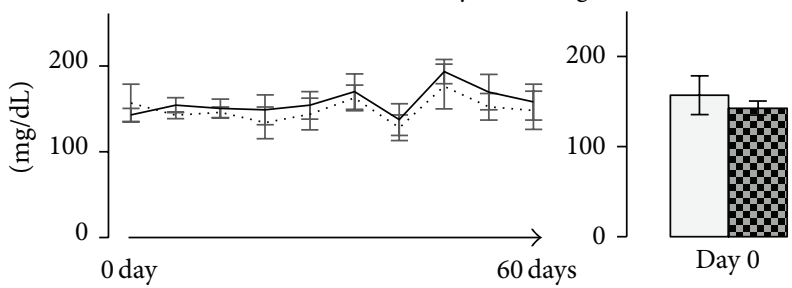

Day 0

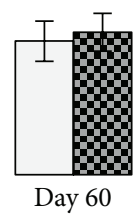

..... Control

$\square$ Control

Metformin

(b)
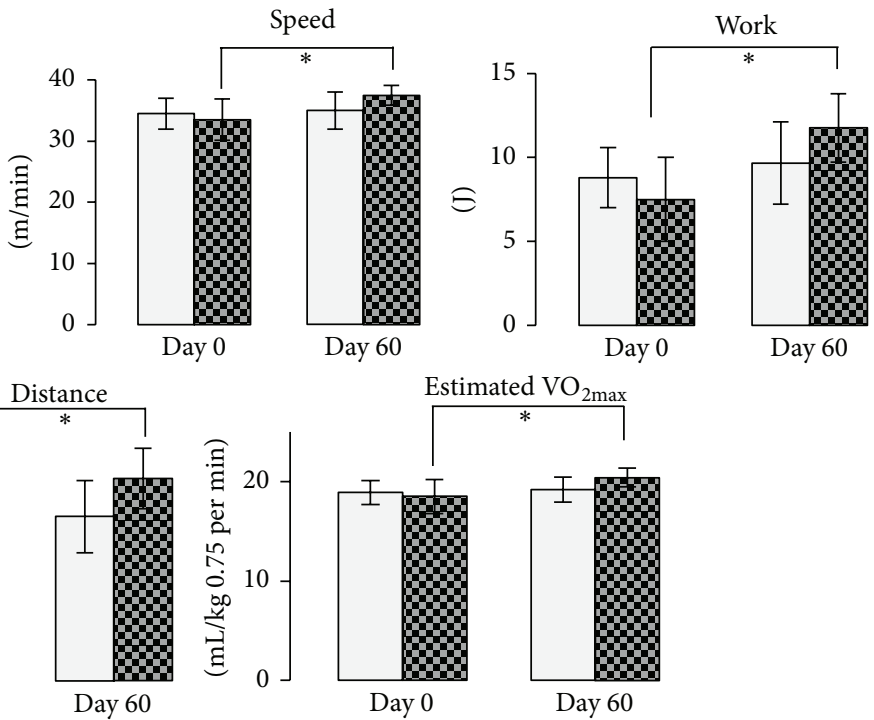

Day 0

Day 60 $\square$ Control
Metformin

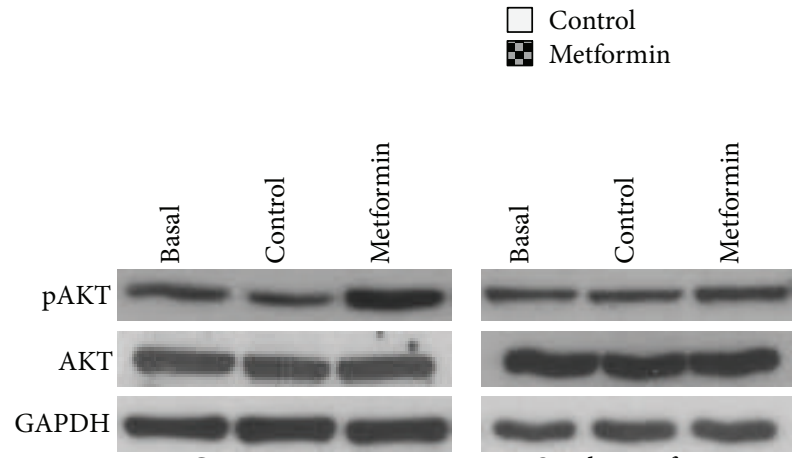

Gastrocnemius

Quadriceps femoris $\square$ Control

Metformin

(c)

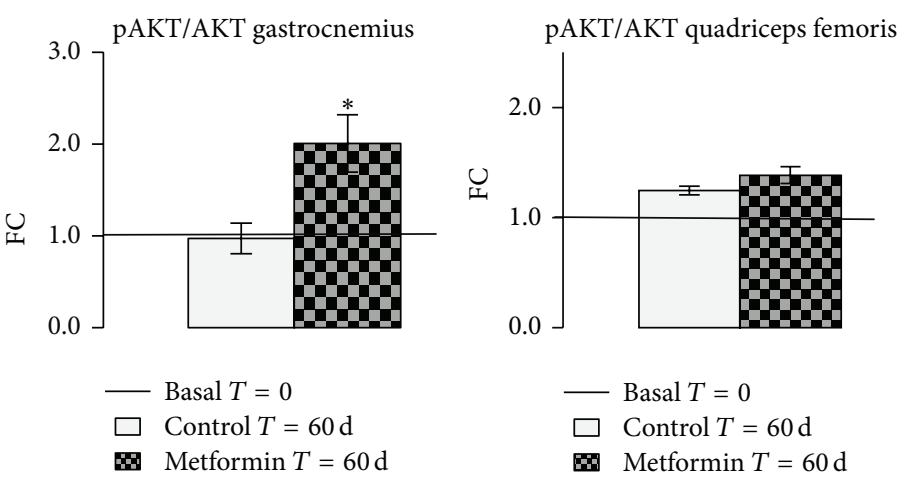

(d)

FIgure 1: Continued. 


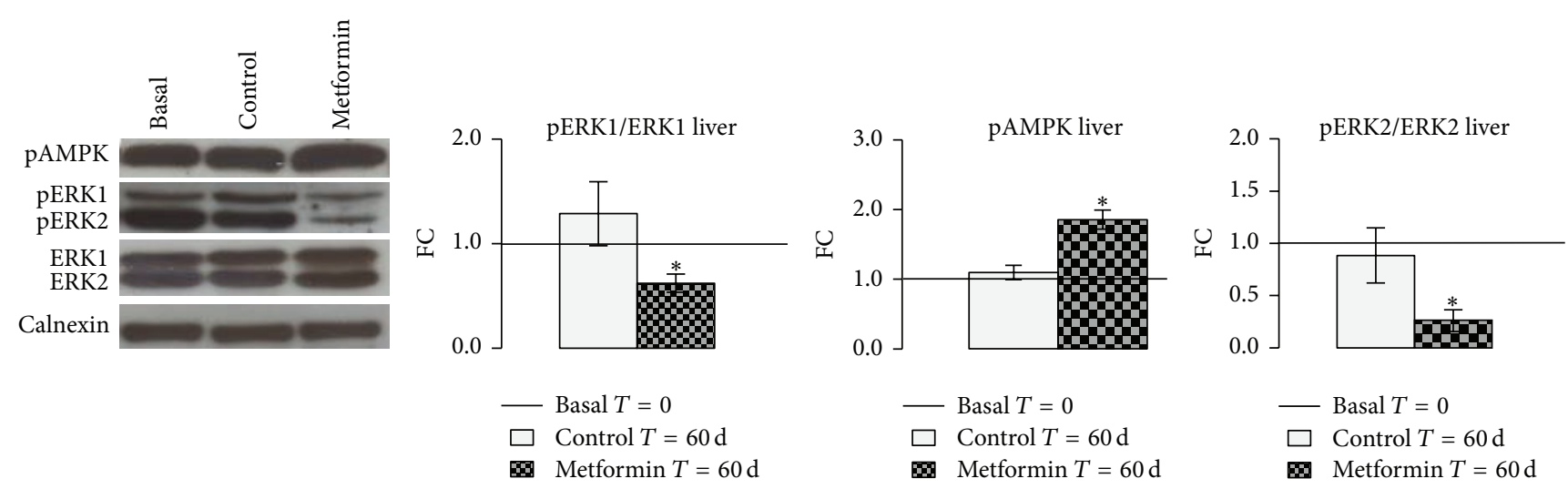

(e)
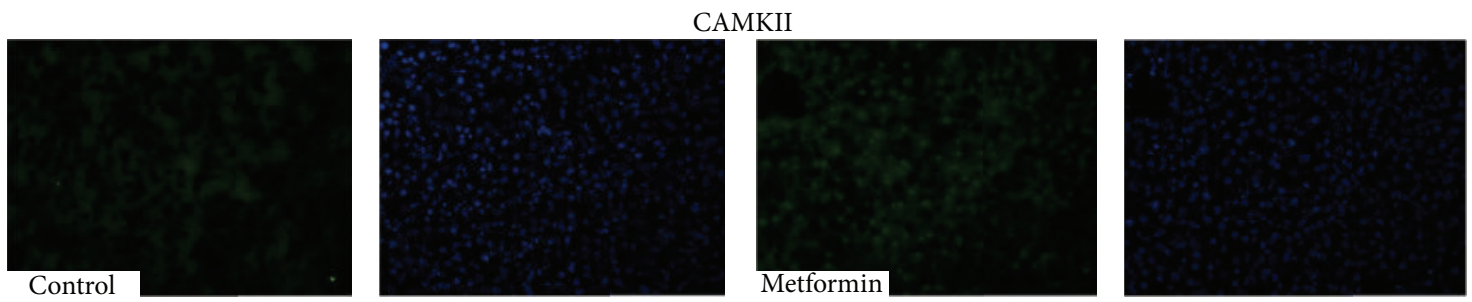

(f)

Figure 1: METF prevention of sedentariness induced damage in adult male mice C57BL/6. (a) Experimental protocol. (b) Weight and glycemia variation. (c) Muscular performance evaluation reveals that METF treatment ameliorated the muscular performance. (d) Muscle tissue analysis indicated that METF had a positive action on AKT activation. (e) Western blot liver analysis showed that METF increased AMPK activation and decreased ERKs activation. (f) Liver morphological studies: METF increased CAMKII signal. Representative immunoblots of analyzed proteins are shown. Scale bar $200 \mu \mathrm{m}$. Significance: ${ }^{*} p \leq 0.05$.

2.3.1. Growth Curve and Cell Viability Test. To study METF action on $\mathrm{C} 2 \mathrm{C} 12$ myoblast proliferation, we performed growth curve assay as described [20]. Briefly, C2C12 myoblasts were plated in $60 \mathrm{~mm} \times 15 \mathrm{~mm}$ culture dishes at $40 \%$ confluence and grown in GM with or without METF and in DM. Medium was changed every $24 \mathrm{~h}$ and the experiment lasted until control cells achieved $70 \%$ of confluence (3 days). Every day, the cells were trypsinized, stained with trypan blue, and were counted using a hemocytometer and the average values for each single day were used to plot a growth curve. Cell viability was calculated by dividing the nonstained viable cell count by the total cell count. In addition, morphological changes were examined daily.

2.4. Western Blot Analysis. Protein extracts, performed as described elsewhere [21], were obtained from mouse tissues or cell cultures by using the following lysis buffer containing: $50 \mathrm{mM}$ Tris/HCl, pH 7.4, $150 \mathrm{mM} \mathrm{NaCl}, 1 \%$ Triton X-100, $1 \mathrm{mM}$ sodium orthovanadate $\left(\mathrm{Na}_{3} \mathrm{VO}_{4}\right), 1 \mathrm{mM}$ EDTA, $1 \mathrm{mM}$ PMSF, $1 \mathrm{mg} / \mathrm{mL}$ aprotinin, $1 \mathrm{mg} / \mathrm{mL}$ leupeptin, and $1 \mathrm{mg} / \mathrm{mL}$ pepstatin.

Aliquots of $30 \mu \mathrm{g}$ supernatant proteins, quantified using Bradford method, were resolved on SDS-PAGE gel and transferred onto nitrocellulose membrane (Protran, Whatman Schleicher \& Schuell). The membranes were incubated with specific primary antibodies and then with HRP conjugated anti-species-specific secondary antibodies.
To confirm equal protein loading per sample, we used antibody anti-calnexin or anti-GADPH. Quantitative measurement of immunoreactive bands intensities, visualized by an enhanced chemiluminescence method (Amersham Pharmacia Biotech, Piscataway, NJ, USA), was performed by densitometric analysis using the Scion Image software (Scion Corporation, Frederick, MD, USA). Data were then converted into fold-changes (FC) of the controls [21].

2.5. Immunofluorescence Analysis. For tissues analysis, $7 \mu \mathrm{m}$ frozen cryosections was fixed in Formaldehyde 4\% overnight at 4C. After that, slides were washed in PBS and incubated for 1 hour at room temperature with $10 \%$ horse serum in PBS with $0.05 \%$ Triton X-100 to block nonspecific binding sites, while $\mathrm{C} 2 \mathrm{C} 12$ cells, fixed and permeabilized as described [22], were blocked with PBS containing $1 \%$ bovine serum albumin. Slides or cells were then immunostained with specific antibodies rhodamine-conjugated and nuclei revealed with DAPI staining. Slides were mounted with Moviol. Cells were observed using Nikon Eclipse 50I microscopy and images were captured using Nis-Elements D 4.00 software (Nikon Instruments Europe BV, Netherlands). Data were displayed and analyzed using Adobe Photoshop CS4. Live C2C12 cells were examined and images were acquired by phase contrast microscopy using the same microscope and digital system described above. 
2.6. Statistical Analysis. All experiments were performed three times. Data are presented as the mean \pm SD. Statistical significances were calculated using $t$-tests. Results were considered significant when $p \leq 0.05$.

\section{Results}

3.1. METF Prevention of Sedentariness Induced Damages in In Vivo METF Experiment. Previous data [8,9] suggested the capacity of METF to extend lifespan in the nematode $C$. elegans and in middle-aged male C57BL/6 mice, improving healthspan mice.

We aimed to investigate the possible role of METF in the prevention of sedentariness induced damages. To achieve this scope, 12-week-old male C57BL/6 mice were chosen, maintained in a condition of total absence of exercise, and treated for 60 days with METF, added in water at the dose of $250 \mathrm{mg} / \mathrm{kg}$ body weight per day (Figure 1(a)).

We did not observe a significant modification in body weight between mice randomized to receive METF and those that did not receive METF (Figure 1(b)). A weight reduction was observed in both groups, comparing the beginning and the end of the experiment. In contrast, we did not observe significant effect in glycemia levels (Figure 1(b)).

METF effect on muscle performance evaluation revealed that METF treatment increased speed, time, work, and estimated maximal oxygen consumption $\left(\mathrm{VO}_{2 \max }\right)$ of acute submaximal incremental exercise in the METF group as compared to their baseline values (Figure 1(c)). Overall, muscular performance capacity was therefore increased in the METF group with respect to the pretreatment condition, despite aging and despite the lack of previous exercise training performed whatsoever.

We evaluated METF effects on AKT. The AKT/mTOR pathway, crucial regulator of skeletal muscle mass, is upregulated during hypertrophy and downregulated during muscle atrophy $[23,24]$. In gastrocnemius muscle of METF treated animals, AKT activation improved compared with controls (Figure $1(\mathrm{~d})$ ). In addition, a trend of an AKT activation was detected in quadriceps femoris muscle in METF treated mice (Figure 1(d)).

Next, we examined METF action on liver (Figure 1(e)). As already described in literature [25], METF increased AMPK activation in liver. Liver ERKs activation is associated with oxidative stress and metabolic dysfunctions, main features of obesity and diabetes [26]. Interestingly, ERKs activation was significantly decreased in liver of METF treated mice (Figure 1(e))

Immunofluorescence analysis (Figure 1(f)) showed how METF positively modulates $\mathrm{Ca}^{2+} /$ calmodulin dependent protein kinase (CAMKII) protein levels in liver tissue with respect to controls, suggesting an important role of METF in cellular calcium homeostasis.

All together, our in vivo data indicated that METF could ameliorate not only age induced damage but also sedentariness induced injury.
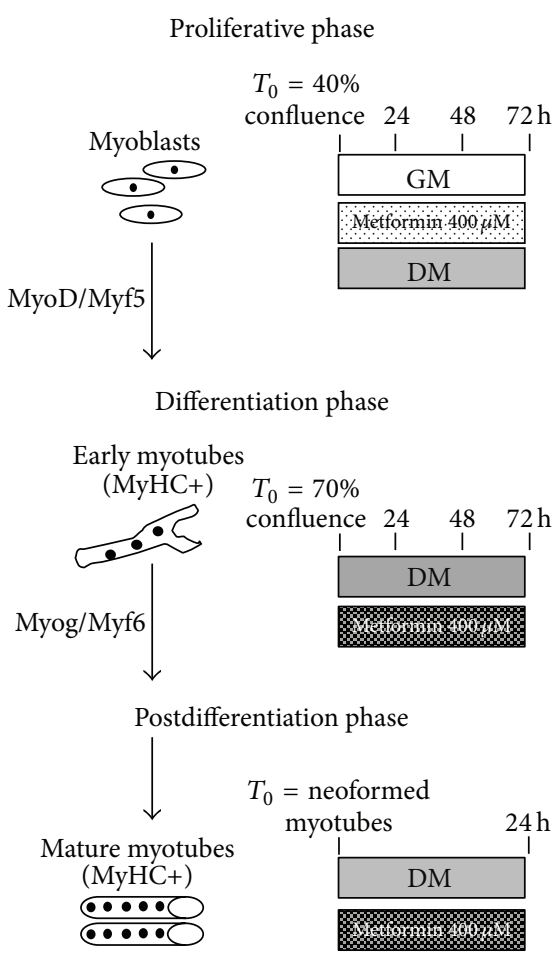

FIgURE 2: Experimental protocol in vitro studies. C2C12 cells in proliferative phase, in differentiation, and after differentiation were treated with $400 \mu \mathrm{M}$ METF.

3.2. METF Positive Role in Proliferation, Differentiation, and Hypertrophy in Skeletal Muscle Myoblasts. We also tested METF action on skeletal muscle proliferation, differentiation, and hypertrophic process, using a C2C12 cell lines (Figure 2). C2C12 murine immortalized cell line provides a good in vitro model for the study of the major steps of myoblasts proliferation and differentiation $[27,28]$. During C2C12 myogenic phenotype achievement, Myogenic Regulator Factors (MRFs) are expressed in a defined sequence; MyoD and Myf5 are primarily expressed, while Myogenin expression is only induced upon muscle differentiation [27, 28].

$400 \mu \mathrm{M}$ METF did not alter C2C12 proliferative potential and did not induce cytotoxic effects, as shown in Figure 3(a) and confirmed in phase contrast images in Figure 3(b). We also measured MyoD protein level. METF led to a significant rise in MyoD content, similarly to DM, compared with control (Figure 3(b)).

Immunofluorescence analysis during proliferation phase revealed that METF increased the protein expression of two key markers of early differentiation: Myf5 and MyoD, suggesting an important role in differentiation induction and promotion of the myoblast commitment to myotube (Figure 3(d)). To confirm this, Phalloidin assay (Figure 3(e)) showed that the cells lost their characteristic circular shape, typical of the active proliferation phase, to achieve a new elongated morphology.

We explored the METF effects on myotube formation. We analyzed METF action on differentiation (Figure 2), from the first phase of differentiation induction $(24 \mathrm{~h})$, middle phase 

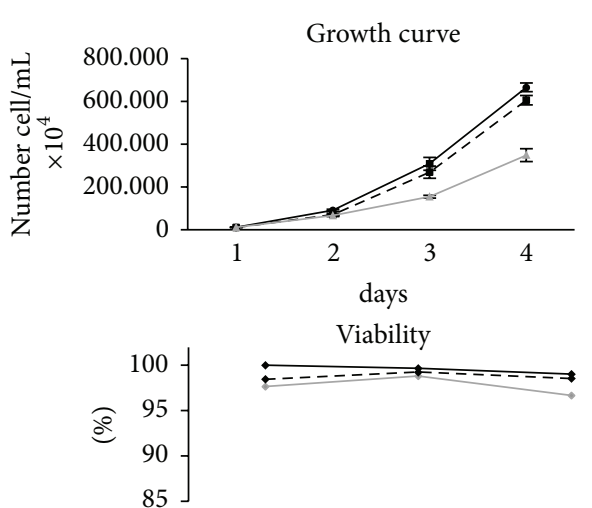

$\rightarrow$ GM $\rightarrow \mathrm{DM}$

(a)
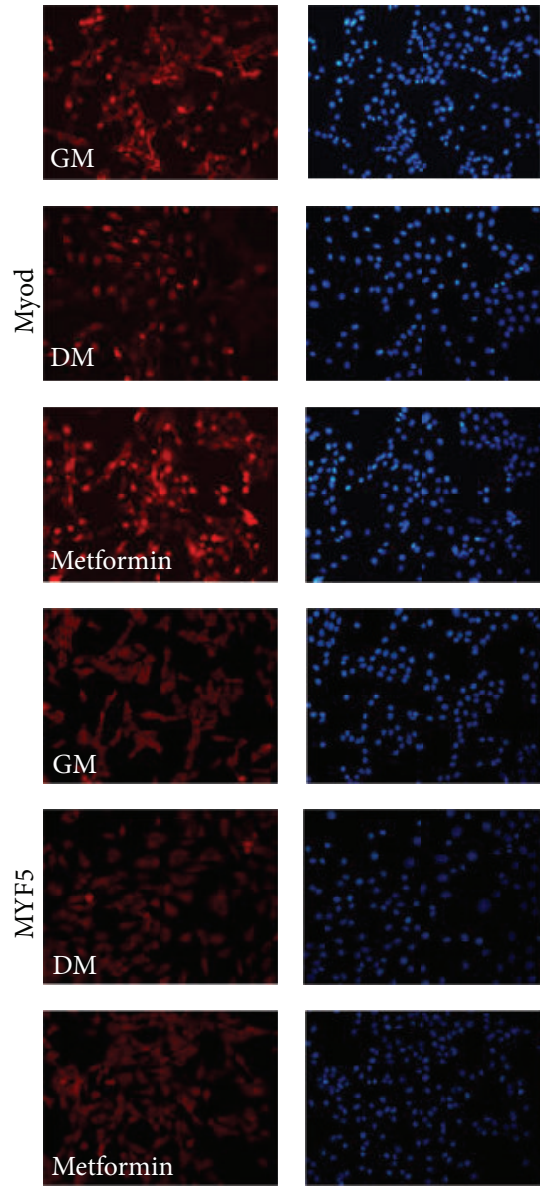

(d)

$24 \mathrm{~h}$ proliferation
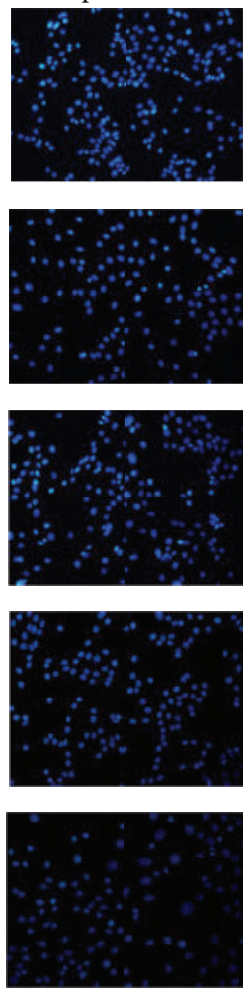

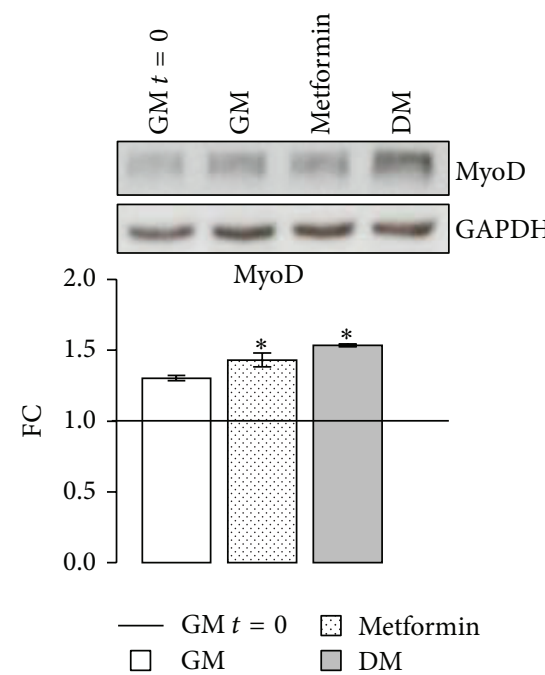

(b)
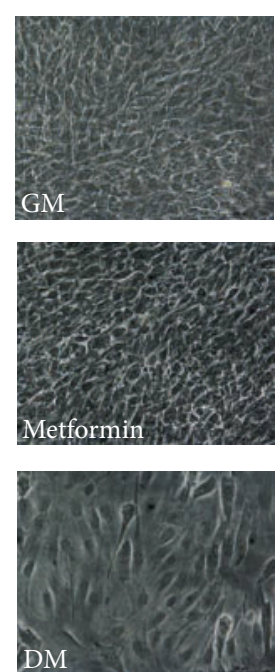

(c)
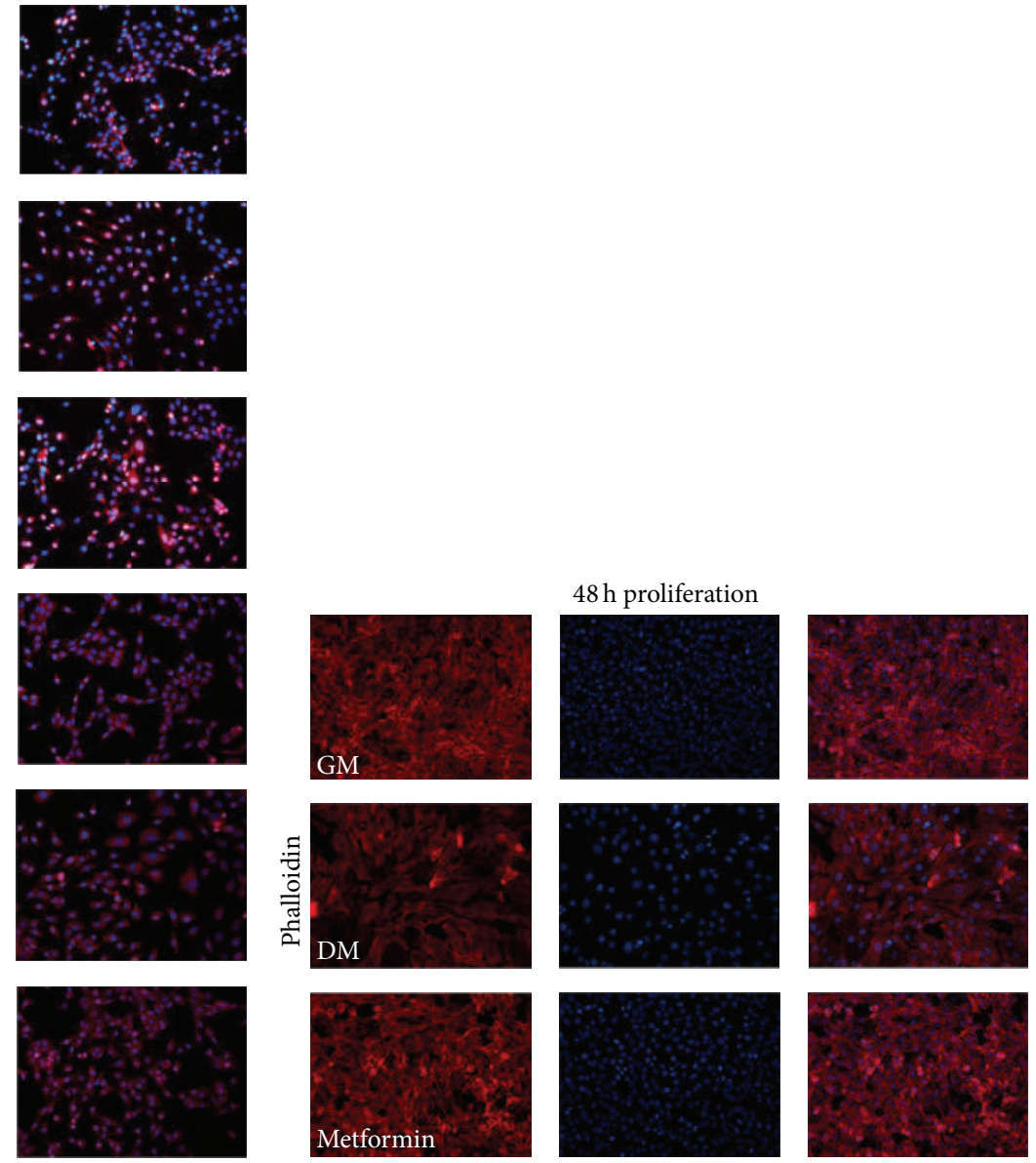

(e)

FIGURE 3: METF action on C2C12 proliferation phase. (a) METF did not modify C2C12 growth rate and did not induce cytotoxic effects. (b) METF increased MyoD protein content with respect to the control. (c) Phase contrast images at the end of proliferation phase. (d) Immunofluorescence analysis of early MRFs MyoD and Myf5 (24 h): METF enhanced myocytes committed. (e) Phalloidin assay at $48 \mathrm{~h}$ of proliferation. Representative immunoblots of analyzed proteins are shown. Scale bar $200 \mu \mathrm{m}$. Significance: ${ }^{*} p \leq 0.05$. 


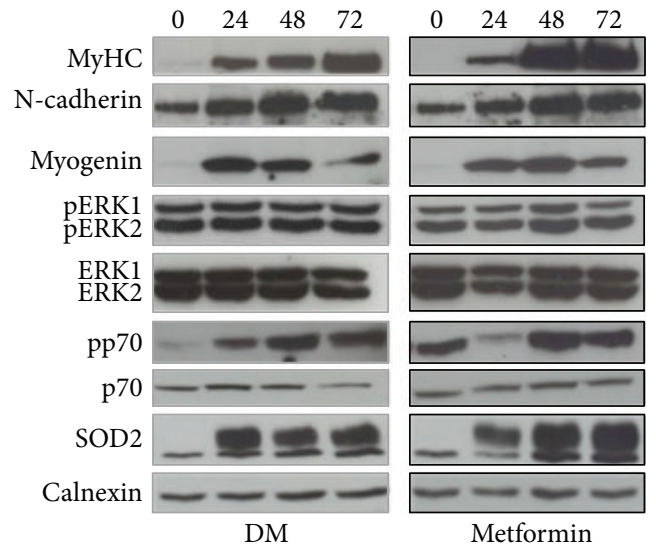

(a)

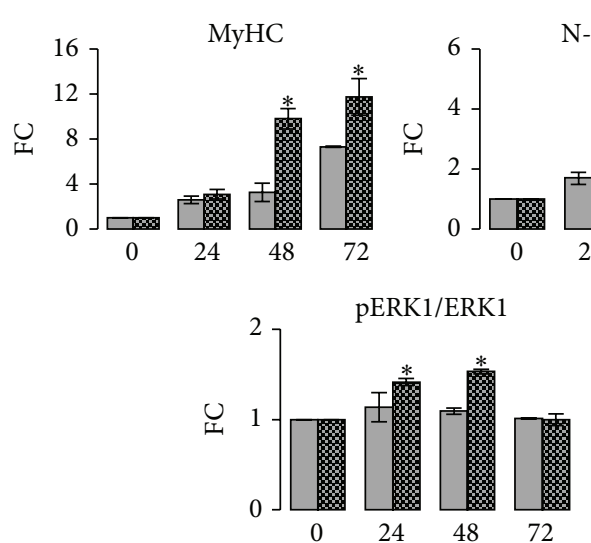

(h)
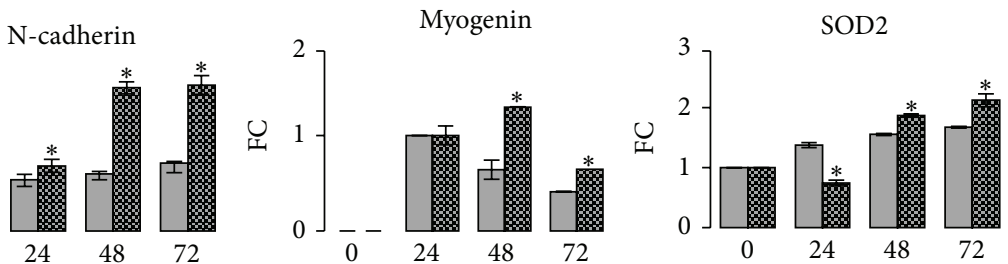

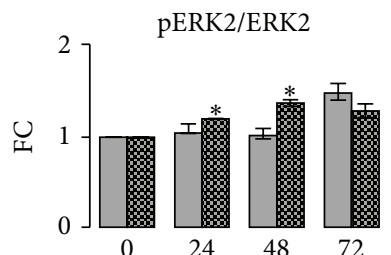

(h)

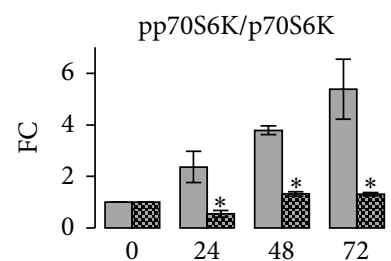

(h)

\section{$\square \mathrm{DM}$}

叉 Metformin

$$
\square \text { DM }
$$

2. Metformin

$\square \mathrm{DM}$

(b)
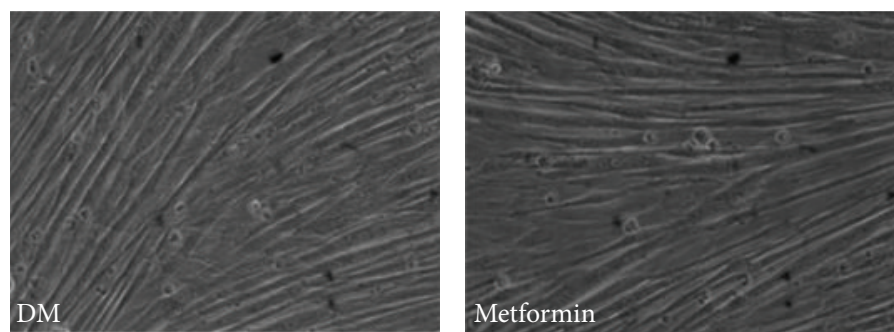

(c)

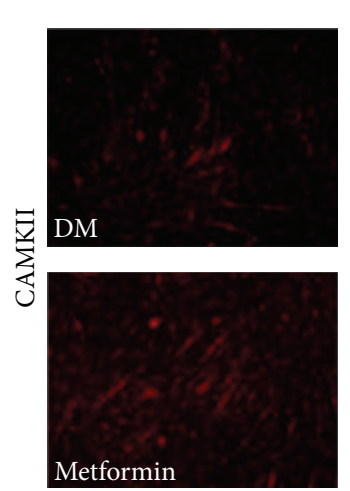

$48 \mathrm{~h}$ differentiation
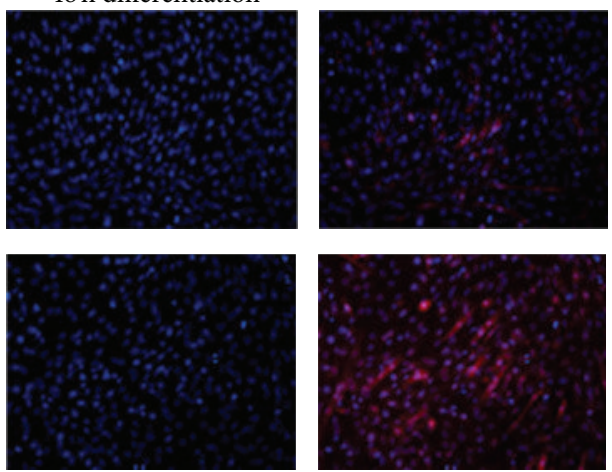

(d)

FIgURE 4: Continued. 


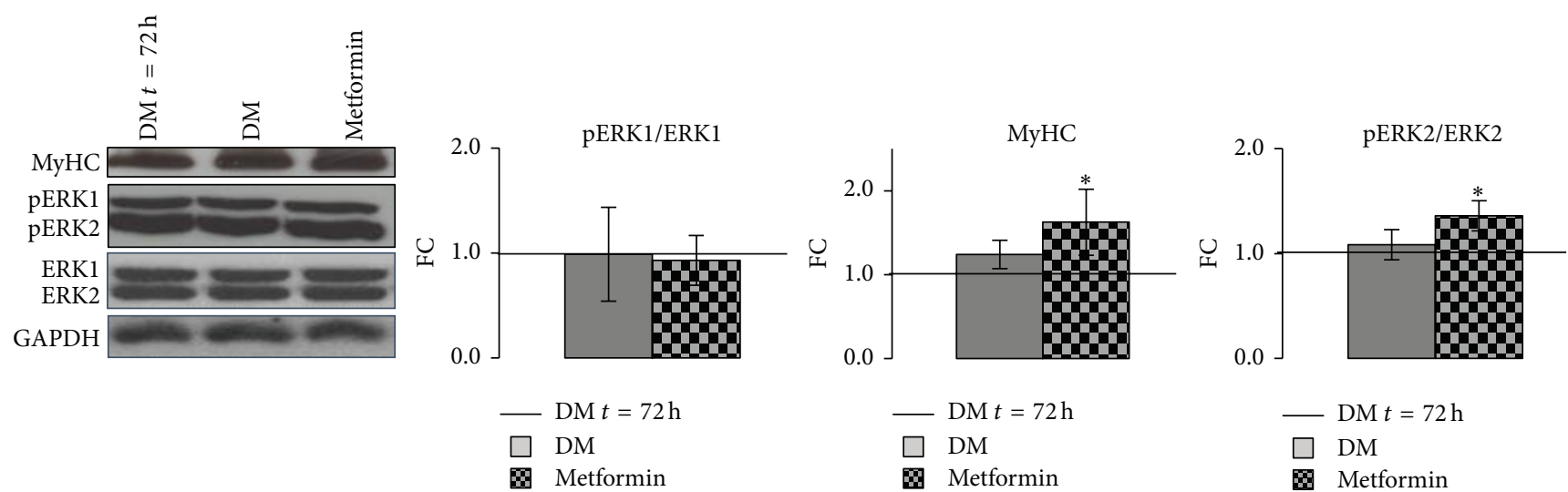

(e)
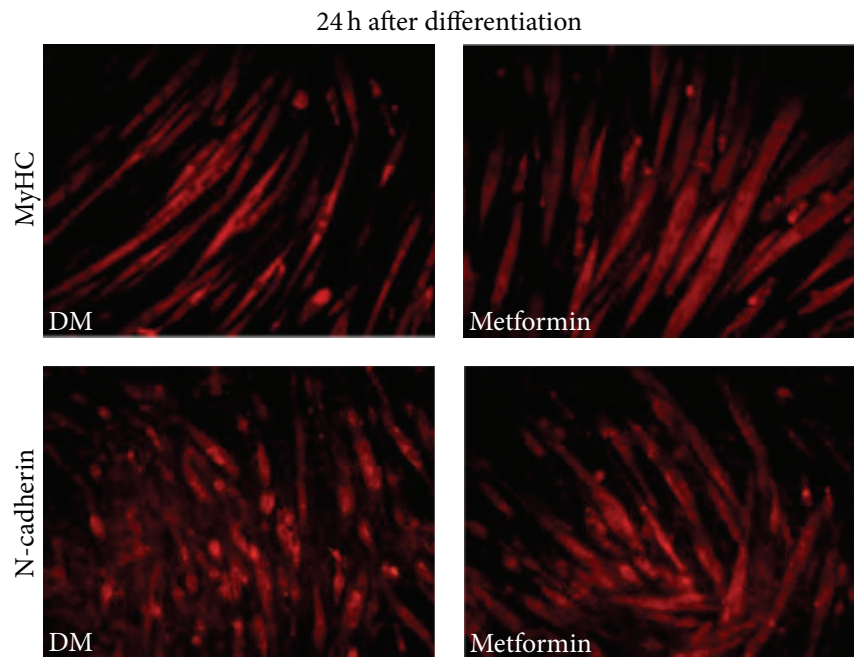

(f)

Figure 4: METF action on C2C12 differentiation and neoformed myotubes. (a) Representative Western blots for C2C12 cell lysates and the indicated antibodies. (b) METF enhanced myotube formation rising MyHC, Myogenin, and N-cadherin protein levels, improved mitochondrial antioxidant proprieties SOD2, activated ERK, and deactivated p70 signaling pathways. (c) Phase contrast images showed that differentiation efficacy is optimal in our model, since all competent myocytes became multinucleated myotubes. (d) At $48 \mathrm{~h}$ from differentiation induction, immunofluorescence analysis indicated positive role of METF in CAMKII protein. (e) METF action on neoformed myotubes: METF improved MyHC protein content and activated ERKs. (f) Immunofluorescence studies confirmed Western blot data of METF action in hypertrophic process. Scale bar $200 \mu \mathrm{m}$. Significance: ${ }^{*} p \leq 0.05$.

$(48 \mathrm{~h})$, and to the end of the process $(72 \mathrm{~h})$, when all fusioncompetent myocytes can form multinucleated myotubes [28]. As shown in Figure 4(b), METF treated cells were characterized by a significant rise in principal marker of myotube maturation, Myosin Heavy Chain (MyHC) protein levels with respect to control cells. The similar METF positive action was observed for $\mathrm{N}$-cadherin, a central cytoskeletal protein involved in cytoskeletal rearrangement, required to the fusion of myoblast in new myotubes [29]. To corroborate our results, which indicated the active role of METF in differentiation progression, we analyzed Myogenin protein levels. METF enhanced Myogenin protein content and, in particular, its expression peak at $48 \mathrm{~h}$ (Figure $4(\mathrm{~b})$ ).

Protein level of Superoxide Dismutase (SOD2), an enzyme that efficiently converts superoxide to the less reactive hydrogen peroxide, was significantly increased in METF cells compared to control cells (Figure 4(b)). This result suggests that METF could counteract the damage caused by a sedentary lifestyle by strengthening the antioxidants mitochondrial functions.

Finally, we investigated METF action on the principal signaling cascades involved in skeletal muscle formation: ERKs and p70S6 kinase pathways [23]. METF enhanced differentiation process through ERKs activation, while it decreased p70S6 kinase pathway (Figure 4(b)).

After $48 \mathrm{~h}$ from differentiation induction, immunofluorescence analysis revealed that CAMKII protein expression was increased in neoformed myotubes treated with METF (Figure 4(d)).

Based on our previous data on effect of short time METF stimulation on the hypertrophic process [10], we studied the effect of long time METF treatment on neoformed 
myotubes (Figure $4(\mathrm{c})$ ). $24 \mathrm{~h}$ of METF stimuli significantly increased MyHC protein levels (Figure 4(e)). As observed in all differentiation phases, METF effects were mediated by ERKs signaling pathways activation (Figure 4(e)).

Also in immunofluorescence images, we observed an important increase in $\mathrm{MyHC}$ and $\mathrm{N}$-cadherin protein content after METF treatment on neoformed myotubes. Furthermore, METF treatment caused important morphological changes in terms of morphological parameters (myotubes length and diameter as shown in Figure 4(f)).

\section{Discussion}

The effects of physical exercise have relevant implications in the prevention and treatment of damage induced by "obesogenic environment" characterized by a positive balance between energy intake and energy expenditure. Moreover, physical activity represents a primary goal in the treatment of diabetes and obesity complications, in particular in skeletal muscle system. To corroborate the fundamental role of physical activity, the World Health Organization has identified physical inactivity as the fourth-leading risk factor for global mortality [30].

We investigated the potential effects of in vivo treatment with METF, currently candidate drug for lifespan extension, on the prevention of sedentariness induced damages. Specifically, we analyzed the METF capacity to improve mouse muscle physical performance.

The study of a protocol of exercise training in mice (Figure 1(c)) indicated that METF could have positive effects on muscular performance. Our results, obtained from studying a model of sedentary healthy mice, confirm the positive METF action on skeletal muscle function previously obtained in older mice models [8].

Several recent works suggest how this biguanide drug could be used in the prevention of aging induced damages $[8,9,31]$. In this perspective, we studied the key molecular regulators involved in common aging pathways [32]. AKT signaling is central in the regulation of muscle function and, in particular, AKT inactivation is associated with muscle damages induced by aging $[23,33]$. We observed that METF increased the activation of AKT in gastrocnemius and quadriceps femoris muscles (Figure $1(\mathrm{~d})$ ), suggesting a hypothetical novel use of this drug not only in agingrelated conditions, but also in sedentary-related damaged muscle conditions. Precisely, for the first time, our work showed how the beneficial effects of METF occur not only in groups already characterized by pathological conditions (e.g., obesity, diabetes, and aging-related disorders) but also in healthy sedentary populations.

An additional positive action was observed in METF treated mouse liver; METF deactivates ERK and promotes CAMKII signaling. ERK activation is crucial in favoring the development of several liver dysfunctions, such as liver fibrosis and hepatocellular cancer [34], while CAMKII pathways activation is fundamental to preserve liver functions [35]. From these data, it is reasonable to conclude that METF supplementation could be utilized to keep liver healthy.
We demonstrated in healthy humans that constant aerobic physical exercise is the clue to avoid lipid steatosis [36].

To further clarify METF cellular mechanisms underlying the effects obtained in the mouse model, we studied METF action using an in vitro model of myoblasts, $\mathrm{C} 2 \mathrm{C} 12$ cell line $[27,28]$. This cell line represents the gold standard of immortalized cells to study not only myogenesis but also the hypertrophy process and its use has allowed us to investigate METF action on cellular pathways involved in muscle training, characteristic process of healthy subject.

First, we observed that METF did not modify $\mathrm{C} 2 \mathrm{C} 12$ proliferation rate and viability (Figure $3(\mathrm{a})$ ), confirming the possible use of this biguanide drug without side effects on skeletal muscle [37]. Those results have important implications since several tumors are associated with sarcopenia and cachexia [38], which might benefit from METF treatment.

METF accelerated myogenic phenotype acquisition and myotubes formation (Figures 3 and 4). After $24 \mathrm{~h}$ of METF stimuli on neomyotubes, we observed an increment in morphological parameters (Figure 4(f)), similar to our previous work where on acute METF treatment was administered [10]. This effect is mediated by ERK activation (Figure 4(e)). Our data indicate that METF should not be considered not only a drug capable of inactivating the cellular mechanisms related to muscle injury, but also a drug capable of activating cellular processes related to muscle strengthening and hypertrophy. We speculate that this METF capacity to enhance myotubes formation and hypertrophy could represent a possible explanation of data obtained, in vivo, in mice.

Finally, our results obtained during differentiation phases showed that SOD2 protein content is increased after METF stimuli (Figure 4(b)). The intracellular enzymes of the SOD family act as a primary line of defense to cope with the deleterious effects of ROS, thereby contributing to an overall decrease in oxidative damage. Lower SOD activity is associated with sedentary lifestyle, characterized by insulin resistance, suggesting that reduced capacities of antioxidant enzymes lead to increased oxidative stress in diabetes and obesity [39]. So, we hypothesize that METF could also act as an antioxidant agent. This potential effect needs further investigation.

\section{Conclusions}

In conclusion, our study reports several novel findings regarding the use of METF in a condition of absence of physical activity and specifically: (1) it improves mice physical aerobic performance; (2) it ameliorates myotubes formation, regulating the principal molecular mediators of skeletal muscle hypertrophy and atrophy; (3) it prevents oxidative stress damage, modulating ERK and SOD signaling.

The relevance of our results resides in a potential use of METF (or drugs with similar biological proprieties) to counteract the damages consequent to sedentariness either directly (acting on molecular targets involved in stress condition) or indirectly, by enhancing the known beneficial physical activity effects. In this framework, additional research is necessary, also in humans, to test combined therapeutical 
use of METF, exercise, and diet to prevent damages of sedentariness.

\section{Conflict of Interests}

No potential conflict of interests was reported.

\section{Authors' Contribution}

Pamela Senesi and Anna Montesano contributed equally to this work. Pamela Senesi, Anna Montesano, and Roberto Codella designed and performed experiments, analyzed data, and wrote the paper. Livio Luzi and Stefano Benedini contributed to the discussion and reviewed the paper. Ileana Terruzzi supervised, designed experiments, and wrote the paper. Ileana Terruzzi is the guarantor of this work and takes responsibility for the integrity of data and the accuracy of data analysis. All authors read and approved the final paper.

\section{References}

[1] E. Ginter and V. Simko, "Type 2 diabetes mellitus, pandemic in 21st century," Advances in Experimental Medicine and Biology, vol. 771, pp. 42-50, 2012.

[2] C. K. Roberts, A. L. Hevener, and R. J. Barnard, "Metabolic syndrome and insulin resistance: underlying causes and modification by exercise training," Comprehensive Physiology, vol. 3, no. 1, pp. 1-58, 2013.

[3] S. Guo, "Insulin signaling, resistance, and metabolic syndrome: insights from mouse models into disease mechanisms," Journal of Endocrinology, vol. 220, no. 2, pp. T1-T23, 2014.

[4] R. Gougeon, "Insulin resistance of protein metabolism in type 2 diabetes and impact on dietary needs: a review," Canadian Journal of Diabetes, vol. 37, no. 2, pp. 115-120, 2013.

[5] E. G. Wilmot, C. L. Edwardson, F. A. Achana et al., "Sedentary time in adults and the association with diabetes, cardiovascular disease and death: systematic review and meta-analysis," Diabetologia, vol. 55, no. 11, pp. 2895-2905, 2012, Erratum in: Diabetologia, vol. 56, no. 4, pp. 942-943, 2013.

[6] C. Y. Jeon, R. P. Lokken, F. B. Hu, and R. M. van Dam, "Physical activity of moderate intensity and risk of type 2 diabetes: a systematic review," Diabetes Care, vol. 30, no. 3, pp. 744-752, 2007.

[7] L. M. Berstein, "Metformin in obesity, cancer and aging: addressing controversies," Aging, vol. 4, no. 5, pp. 320-329, 2012.

[8] A. Martin-Montalvo, E. M. Mercken, S. J. Mitchell et al., "Metformin improves healthspan and lifespan in mice," Nature Communications, vol. 4, article 3192, 2013.

[9] B. Onken and M. Driscoll, "Metformin induces a dietary restriction-like state and the oxidative stress response to extend C. elegans healthspan via AMPK, LKB1, and SKN-1," PLoS ONE, vol. 5, no. 1, Article ID e8758, 2010.

[10] I. Terruzzi, P. Senesi, C. Magni et al., "Insulin-mimetic action of conglutin- $\gamma$, a lupin seed protein, in mouse myoblasts," Nutrition, Metabolism \& Cardiovascular Diseases, vol. 21, no. 3, pp. 197-205, 2011.

[11] B. Strasser and D. Pesta, "Resistance training for diabetes prevention and therapy: experimental findings and molecular mechanisms," BioMed Research International, vol. 2013, Article ID 805217, 8 pages, 2013.
[12] D. G. Candow, S. C. Forbes, J. P. Little, S. M. Cornish, C. Pinkoski, and P. D. Chilibeck, "Effect of nutritional interventions and resistance exercise on aging muscle mass and strength," Biogerontology, vol. 13, no. 4, pp. 345-358, 2012.

[13] S. C. Forbes, J. P. Little, and D. G. Candow, "Exercise and nutritional interventions for improving aging muscle health," Endocrine, vol. 42, no. 1, pp. 29-38, 2012.

[14] J. E. Ostler, S. K. Maurya, J. Dials et al., "Effects of insulin resistance on skeletal muscle growth and exercise capacity in type 2 diabetic mouse models," The American Journal of Physiology-Endocrinology and Metabolism, vol. 306, no. 6, pp. E592-E605, 2014.

[15] E. A. Richter and M. Hargreaves, "Exercise, GLUT4, and skeletal muscle glucose uptake," Physiological Reviews, vol. 93, no. 3, pp. 993-1017, 2013.

[16] C. K. Roberts, J. P. Little, and J. P. Thyfault, "Modification of insulin sensitivity and glycemic control by activity and exercise," Medicine and Science in Sports and Exercise, vol. 45, no. 10, pp. 1868-1877, 2013.

[17] C. Algire, M. Zakikhani, M. J. Blouin, J. H. Shuai, and M. Pollak, "Metformin attenuates the stimulatory effect of a high-energy diet on in vivo LLC1 carcinoma growth," Endocrine-Related Cancer, vol. 15, no. 3, pp. 833-839, 2008.

[18] J. A. Calvo, T. G. Daniels, X. Wang et al., "Muscle-specific expression of PPAR $\gamma$ coactivator- $1 \alpha$ improves exercise performance and increases peak oxygen uptake," The Journal of Applied Physiology, vol. 104, no. 5, pp. 1304-1312, 2008.

[19] P. Senesi, L. Luzi, A. Montesano, and I. Terruzzi, "DNA demethylation accelerates myoblasts hypertrophy during the late phase of myogenesis activating the IGF-1 pathway," Endocrine, vol. 47, no. 1, pp. 244-254, 2014.

[20] A. Montesano, L. Luzi, P. Senesi, N. Mazzocchi, and I. Terruzzi, "Resveratrol promotes myogenesis and hypertrophy in murine myoblasts," Journal of Translational Medicine, vol. 11, no. 1, article 310, 2013.

[21] P. Senesi, L. Luzi, A. Montesano, N. Mazzocchi, and I. Terruzzi, "Betaine supplement enhances skeletal muscle differentiation in murine myoblasts via IGF-1 signaling activation," Journal of Translational Medicine, vol. 11, no. 1, article 174, 2013.

[22] A. Montesano, L. Luzi, P. Senesi, and I. Terruzzi, "Modulation of cell cycle progression by 5 -azacytidine is associated with early myogenesis induction in murine myoblasts," International Journal of Biological Sciences, vol. 9, no. 4, pp. 391-402, 2013.

[23] D. J. Glass, "Signalling pathways that mediate skeletal muscle hypertrophy and atrophy," Nature Cell Biology, vol. 5, no. 2, pp. 87-90, 2003.

[24] K.-M. V. Lai, M. Gonzalez, W. T. Poueymirou et al., "Conditional activation of Akt in adult skeletal muscle induces rapid hypertrophy," Molecular and Cellular Biology, vol. 24, no. 21, pp. 9295-9304, 2004.

[25] B. Viollet and M. Foretz, "Revisiting the mechanisms of metformin action in the liver," Annales d'Endocrinologie, vol. 74, no. 2, pp. 123-129, 2013.

[26] S. R. Kimball and L. S. Jefferson, "Induction of REDD1 gene expression in the liver in response to endoplasmic reticulum stress is mediated through a PERK, eIF2 $\alpha$ phosphorylation, ATF4-dependent cascade," Biochemical and Biophysical Research Communications, vol. 427, no. 3, pp. 485-489, 2012.

[27] P. Ferri, E. Barbieri, S. Burattini et al., "Expression and subcellular localization of myogenic regulatory factors during the differentiation of skeletal muscle C2C12 myoblasts," Journal of Cellular Biochemistry, vol. 108, no. 6, pp. 1302-1317, 2009. 
[28] S. Burattini, R. Ferri, M. Battistelli, R. Curci, F. Luchetti, and E. Falcieri, "C2C12 murine myoblasts as a model of skeletal muscle development: morpho-functional characterization," European Journal of Histochemistry, vol. 48, no. 3, pp. 223-233, 2004.

[29] N. Taulet, F. Comunale, C. Favard, S. Charrasse, S. Bodi, and C. Gauthler-Rouvière, "N-cadherin/p120 catenin association at cell-cell contacts occurs in cholesterol-rich membrane domains and is required for RhoA activation and myogenesis," The Journal of Biological Chemistry, vol. 284, no. 34, pp. 23137-23145, 2009.

[30] J.-P. Chaput, É. Doucet, and A. Tremblay, "Obesity: a disease or a biological adaptation? An update," Obesity Reviews, vol. 13, no. 8, pp. 681-691, 2012.

[31] W. de Haes, L. Frooninckx, R. Van Assche et al., "Metformin promotes lifespan through mitohormesis via the peroxiredoxin PRDX-2," Proceedings of the National Academy of Sciences, vol. 111, no. 24, pp. E2501-E2509, 2014.

[32] E. Marzetti, R. Calvani, M. Cesari et al., "Mitochondrial dysfunction and sarcopenia of aging: from signaling pathways to clinical trials," International Journal of Biochemistry \& Cell Biology, vol. 45, no. 10, pp. 2288-2301, 2013.

[33] L. A. Consitt, J. van Meter, C. A. Newton et al., "Impairments in site-specific AS160 phosphorylation and effects of exercise training," Diabetes, vol. 62, no. 10, pp. 3437-3447, 2013.

[34] J.-J. Yang, H. Tao, C. Huang, and J. Li, "Nuclear erythroid 2related factor 2: a novel potential therapeutic target for liver fibrosis," Food and Chemical Toxicology, vol. 59, pp. 421-427, 2013.

[35] L. Ozcan, C. C. L. Wong, G. Li et al., "Calcium signaling through CaMKII regulates hepatic glucose production in fasting and obesity," Cell Metabolism, vol. 15, no. 5, pp. 739-751, 2012.

[36] G. Perseghin, F. D. Cobelli, A. Esposito et al., "Left ventricular function and energy metabolism in middle-aged men undergoing long-lasting sustained aerobic oxidative training," Heart, vol. 95, no. 8, pp. 630-635, 2009.

[37] A. Leone, E. Di Gennaro, F. Bruzzese, A. Avallone, and A. Budillon, "New perspective for an old antidiabetic drug: metformin as anticancer agent," Cancer Treatment and Research, vol. 159, pp. 355-376, 2014.

[38] N. Johns, N. A. Stephens, and K. C. H. Fearon, "Muscle wasting in cancer," The International Journal of Biochemistry \& Cell Biology, vol. 45, no. 10, pp. 2215-2229, 2013.

[39] J. W. Baynes and S. R. Thorpe, "Role of oxidative stress in diabetic complications: a new perspective on an old paradigm," Diabetes, vol. 48, no. 1, pp. 1-9, 1999. 


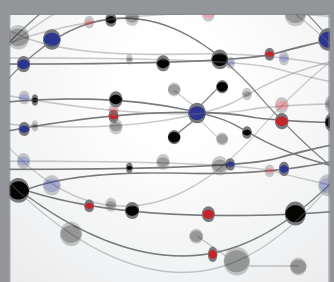

The Scientific World Journal
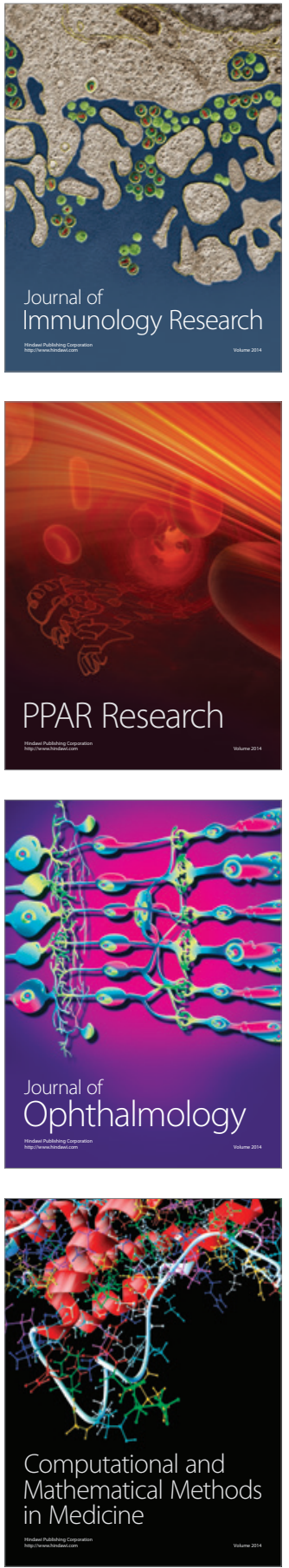

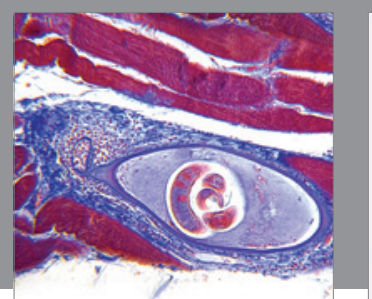

Gastroenterology Research and Practice

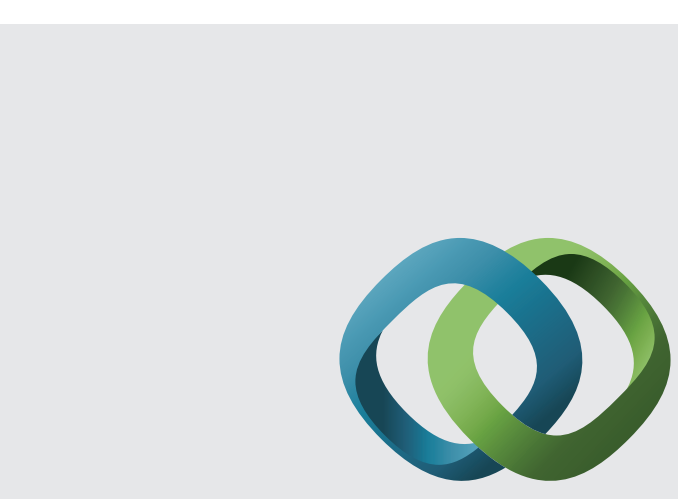

\section{Hindawi}

Submit your manuscripts at

http://www.hindawi.com
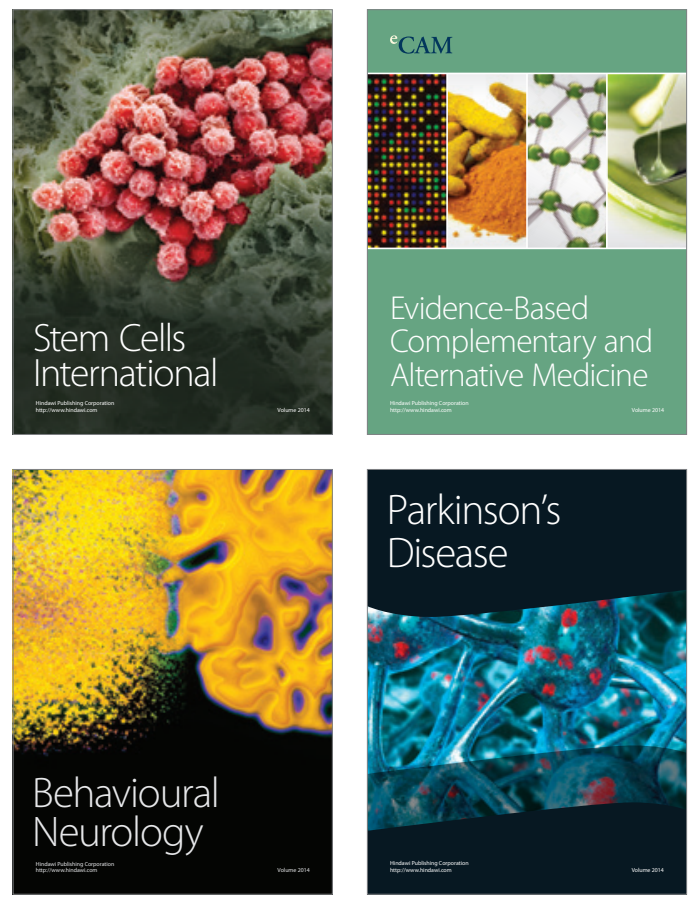
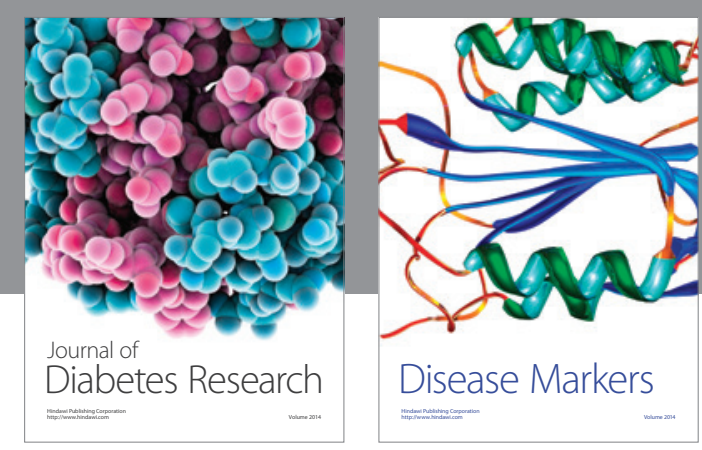

Disease Markers
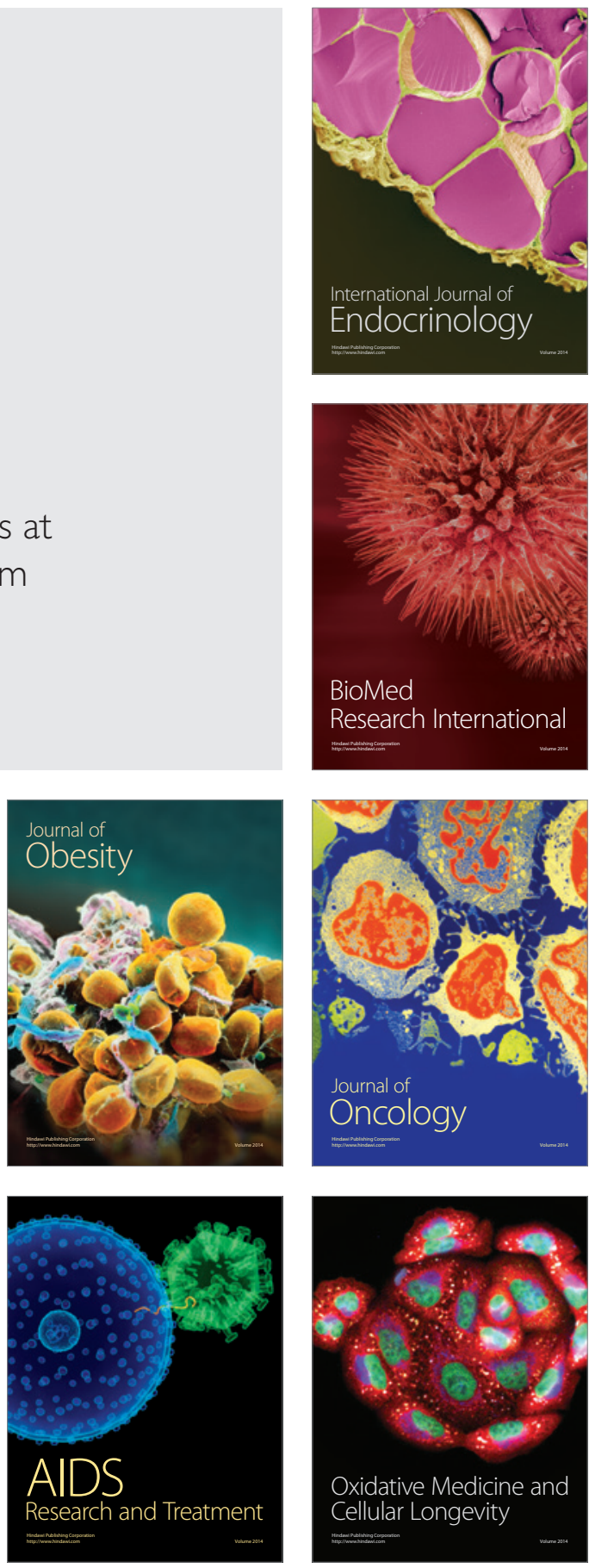